\title{
Variable-interval schedules of token reinforcement
}

\author{
E. F. MALAGODI \\ V. A. HOSPITAL, CORAL GABLES, FLORIDA
}

Two rats received token reinforcement for lever-pressing on VI schedules. Initially, tokens were immediately exchangeable for food. The rats were subsequently required to obtain groups of tokens before they could be exchanged. As the number of tokens required for exchange was gradually increased, response rates for token-production decreased. The variable-interval pattems of responding were disrupted as soon as two tokens were required for exchange.

Several studies by Kelleher (1956, 1957a, b, c, 1958) have investigated the effectiveness of fixed-ratio and fixed-interval schedules of token reinforcers in maintaining responding of chimpanzees in extended behavior sequences. A study by the present investigator (Malagodi, 1967b) investigated the effectiveness of fixed-ratio schedules of token reinforcement in maintaining responding of rats in extended behavior sequences. In the present study investigations were made of extended behavior sequences maintained by variable-interval schedules of token reinforcement. Method

The Ss were two adult male non-agouti brown rats (I-1 and I-6). A detailed description of their experimental histories and of the apparatus has been presented elsewhere (Malagodi, 1967a). The Ss had been trained in a modified Skinner-box experimental chamber which contained a Gerbrands rat lever, a token hopper into which tokens (dark glass marbles) were dispensed, a token receptacle into which the rats deposited the tokens, and a pellet hopper into which $45 \mathrm{mg}$ Noyes standard formula food pellets were dispensed. Automatic programming and recording equipment was located in an adjacent room. The rats were maintained at $80 \%$ of their free-feeding body weights, and were run daily.

In the presence of illumination from a stimulus lamp located above the lever, lever-presses resulted in the delivery of tokens on a 1-min variable-interval schedule of token reinforcement (VI 1 TOK). A brief tone accompanied token delivery. In the presence of localized illumination of the token receptacle and a continuous low-frequency clicking sound, token deposits into the receptacle resulted in the delivery of food pellets on a continuous schedule of primary reinforcement (crf). Initially, the exchange schedule was a one token fixed-ratlo exchange schedule, or FR 1 EX; that 1s, the tokens were exchangeable for food as they were delivered. Then, with Rat I-1, the exchange ratio was gradually increased to a five token variable-ratio exchange schedule, or VR 5 EX. The combined schedule may be viewed as a secondorder schedule of reinforcement (Kelleher, 1966), and designated as VR $5 \mathrm{EX}$ (VI 1 TOK); that is, tokens were delivered on a VI $1 \mathrm{~min}$ schedule and, on the average, five tokens had to be obtained before the tokens could be exchanged for food. With Rat I-6 the schedule of token reinforcement was VI 1 as the exchange ratio was gradually increased to a five token fixed-ratio exchange schedule, or FR 5 EX; the schedule of token reinforcement was then reduced to VI .5 min and the exchange ratio was increased to FR 10 EX.

The number of sessions at each value of the exchange ratios varied between the two rats, and from value to value, and are presented in Table 1.

\section{Results and Discussion}

It may be seen in Table 1 that overall rates of lever-pressing decreased as the exchange ratios were increased. Both Ss made several deposit errors (token deposits prior to the completion of the exchange requirements) on the first few sessions at VR $2 \mathrm{EX}$ or FR $2 \mathrm{EX}$, and thereafter made no further errors. The decreases in overall response rates as a function of increased exchange ratios were not merely reductions in the slopes of the response curves, but were characterized by alternating periods of steady responding characteristic of VI responding, and periods of no responding at all. Representative cumulative records for Rat I-6 under three schedules are presented in Fig. 1. It may be seen in the first record that overall performance is typical of behavior under VI schedules; responding is relatively stable in rate, and there is little pausing. In the second record, which was taken from the fifth session at FR 2 EX, this S tended to respond at typical VI rates until the first token was delivered; following delivery of the first token the rat paused, and most frequently the second token was delivered on the first response following such a pause. Responding under FR $10 \mathrm{EX}$ (VI .5 TOK), in the third record, was similar to responding under FR 2 EX (VI 1 TOK) in that the rat tended to respond

Table 1

Mean response rates as a function of increases

in exchange ratios

\begin{tabular}{lrlllrl} 
& & \multicolumn{2}{c}{ Rat 1-1 } & \multicolumn{2}{c}{ Rot 1-6 } \\
Schedule & Sessions & Rate & Schedule & Sessions & Rate \\
\hline FR 1 EX & 54 & $19.27^{\circ}$ & FR 1 EX & 11 & 13.42 \\
VR 2 EX & 5 & 10.09 & FR 2 EX & 7 & 7.64 \\
VR 3 EX & 5 & 6.99 & FR 3 EX & 1 & 4.62 \\
VR 4 EX & 5 & 7.73 & FR 4 EX & 1 & 3.90 \\
VR 5 EX & 14 & 4.81 & FR 5 EX & 2 & 2.69 \\
& & & FR 10 EX & 14 & 1.72 \\
\hline
\end{tabular}

$a$ based on last 10 sessions. 


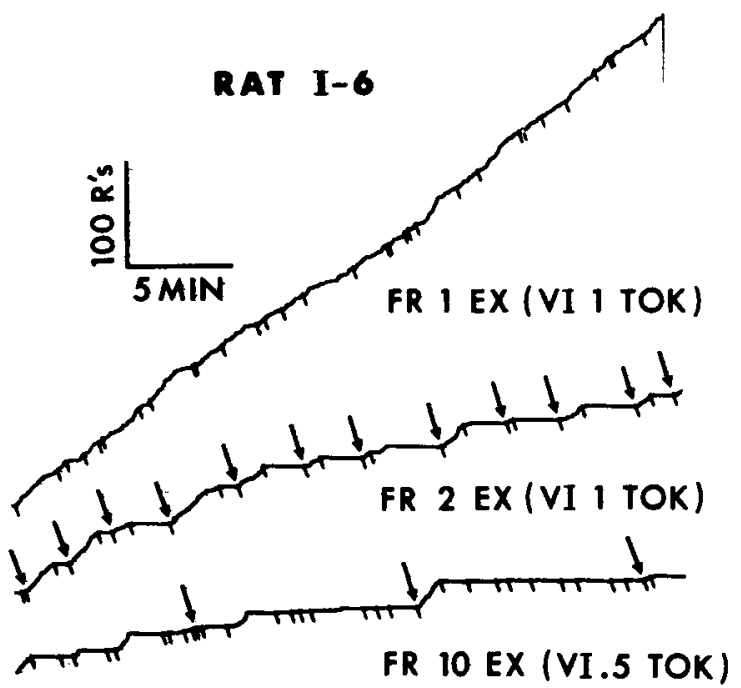

Fig. 1. Representative cumulative records for Rat I-6. Oblique pips indicate token delivery. In the second and third records the points at which the tokens were exchanged for food are indicated by the arrows. The recorder was inoperative during exchange.

at typical VI rates until the first token was delivered, and rarely sustained responding after the first token was delivered. Performance of Rat I-1 followed the same pattern as shown for Rat I-6; the VI patterns of responding that typifled performance under FR 1 EX (VI 1 TOK) were disrupted as soon as the exchange ratio was increased to VR 2 EX.

The results are similar to those found by Kelleher $(1957 \mathrm{~b}, \mathrm{c})$ with fixed-interval schedules of token reinforcement. In one of these studies (Kelleher, 1957c), for example, the chimpanzees stopped responding under FR 8 EX (FI 5 TOK). As Kelleher has noted, the schedules according to which tokens are delivered are of critical importance in determining their effectiveness in maintaining responding in extended behavior sequences. The results of Kelleher's (1956, 1957a, 1958) studies of fixed-ratio schedules of token rein- forcement with chimpanzees and a previous study by the present investigator with fixed-ratio schedules of token reinforcement with rats (Malagodi, 1967b) suggest that ratio schedules are more effective than interval schedules in sustaining such behavior. This is not surprising when one considers the different possibilities of accidental reinforcement that exist under ratio and interval schedules. Post-token pauses in extended fixed-ratio schedules are not followed immediately by token delivery, whereas post-token pauses in extended fixed-interval and variable-interval schedules can be followed by immediate token delivery on the first response following such pauses; these schedule contingencies could differentially reinforce other behavior (e.g., manipulating tokens) which immediately precedes the delivery of token reinforcers.

The results of the present study and of previous studies of token reinforcement with rats (Malagodi, $1967 a, b)$ indicate that laboratory rats may be effectively used as Ss in the token paradigm; these interspecies extensions of behavioral control techniques should allow for more extensive investigations of scheduling and other variables determining the effectiveness of token reinforcers.

\section{References}

Kelleher, R. T. Intermittent conditioned reinforcement in chimpanzees. Science, 1956, 124, 679-680.

Kelleher, R. T. A comparison of conditioned and food reinforcement. in chimpanzees. Psychol. Newsltr., 1957a, 8, 88-93.

Kelleher, R. T. A multiple schedule of conditioned reinforcement with chimpanzees. Psychol. Rep., 1957b, 3, 485-491.

Kelleher, R. T. Conditioned reinforcement in chimpanzees. $J$. comp. physiol. Psychol., 1957c, 49, 571-575.

Kelleher, R. T. Fixed-ratio schedules of conditioned reinforcement with chimpanzees. J. exp. Anal. Behav., 1958, 1, 281-289.

Kelleher, R. T. Chaining and conditioned reinforcement. In $\mathbf{W} . \mathbf{K}$. Honig (Ed.), Operant behavior: areas of research and application. New York: Appleton-Century-Crofts, 1966. Pp. 160-212.

Malagodi, E. F. Acquisition of the token-reward habit in the rat. Psychol. Rep., 1967a, in press.

Malagodi, E. F. Fixed-ratio schedules of token reinforcement. Psychon. Sci., 1967b, in press. 